\title{
Analysis of Sample Rejection and the Impact on Quality of Care in Patients in a Single Tertiary Healthcare Facility in Malaysia
}

\author{
Fatmawati Kamal, Wan Asmuni Wan Mohd Saman, Madyhah Adbul Monir, Mariana Rahmat \\ Department of Pathology, Faculty of Medicine, \\ Universiti Teknologi MARA, Sungai Buloh, Selangor, Malaysia \\ fatmawati.kama@@gmail.com,wan.asmuni@yahoo.com,madyhah.monir@gmail.com, marianarahmat@yahoo.com \\ Tel: $013-6265250$
}

\begin{abstract}
The Malaysian Society for Quality in Health (MSQH), recommended a rejection rate of less than $1 \%$. Many studies reported rejection rates of $0.1 \%$ to $3.49 \%$. This study was conducted at the UiTM Medical Specialist Centre from January 2019 to October 2019. The blood bank and haematology workstation both rejected $84(5.7 \%)$ and $560(3.38 \%)$ samples, respectively. The main causes of sample rejection were clotted and lysed samples. Most of the rejected samples came from the wards, followed by the emergency department. This study showed the need for improvement in venipuncture techniques and types of equipment.
\end{abstract}

Keywords: Rejection rates; Sample rejection; Quality of care; Tertiary centre

eISSN: 2398-4287 @ 2020. The Authors. Published for AMER ABRA cE-Bs by e-International Publishing House, Ltd., UK. This is an open access article under the CC BYNC-ND license (http://creativecommons.org/licenses/by-nc-nd/4.0). Peer-review under responsibility of AMER (Association of Malaysian Environment-Behaviour Researchers), ABRA (Association of Behavioural Researchers on Asians) and cE-Bs (Centre for Environment-Behaviour Studies), Faculty of Architecture, Planning \& Surveying, Universiti Teknologi MARA, Malaysia.

DOI: https://doi.org/10.21834/e-bpj.v5i13.2104

\subsection{Introduction}

Sample rejection are samples rejected by the laboratory if the sample was not obtained as per technical instruction for the specific specimen. Sample rejection is often regarded as a nuisance by everyone, not just the clinical staffs. For clinical staffs, it means having to redo sample collection and sending it back to the laboratory. This can lead to delay in getting the results for patients and thus, might affect patient management such as decision on treatment and delay of hospital discharge, not to mention the pain associated with venipuncture. Laboratory personnel or medical laboratory technologists would have to issue a notice of rejection to the ward or clinic for every rejection. Oftentimes, they also had to inform the ward or clinic regarding the rejection. All details regarding the rejection will have to be filed accordingly to allow adequate analysis of the cause of rejection and possible intervention to improve the quality of care for patients. It has become a quality indicator for accreditation of medical laboratory in Malaysia.

\subsection{Literature Review}

Sample rejection can affect patients' management. Repeated venipuncture causes inconvenience and discomfort for both the patient and the healthcare worker, as it causes pain, consumes time and increases workload. Moreover, it creates a delay in obtaining laboratory result. If the repeated test result is abnormal, it can cause further delay in the commencement of the correct treatment for the patient. Sample rejection can also lead to the abandonment of the test. A study reported that as many as $48.3 \%$ of rejected tests were never being redrawn and reported (Jacobsz, 2011).

Sample rejection is something the laboratory has to do to ensure quality. The laboratory has to make sure that all its results are reliable, accurate and timely. The inaccuracy of the result can be caused by the limitation of the test, machine and operator when making 
a measurement. The rejection rate reflects the preanalytical process of the laboratory path of workflow, which includes sample collection and transport. Studies conducted by different institutions have reported rejection rates of $0.1 \%$ to $3.49 \%$ (Alsina, 2008; Bonini, 2002; Stark, 2007). The Malaysian Society for Quality in Health (MSQH), in its 2017 5th edition of the MSQH Hospital Accreditation Standards, recommended a rejection rate of less than $1 \%$, as one of the quality indicators in the medical laboratories (Malaysian Society for Quality in Health, 2017).

There is no standard method to set the target rejection rate. However, the College of American Pathologists (CAP) suggests that each institution compare its internal rates of specimen rejection to benchmarks from multi-institutional studies. The institution should specify a low threshold for action if the rejection rates increase very much. A 1997 CAP Q-probes studies reported a median rejection rate of $0.31 \%$, while in 2002 , a Q-tracks study reported a rejection rate target of $0.28 \%$ amongst the best performing laboratories (Jones, 1997) (Karcher, 2014). This study also highlighted the practice of redefining benchmarks by the participants in the first two years of CAP Q-tracks program. There were reports from laboratories with excessive rejection rates. However, not many have reported significant improvement. Few have suggested a few methods to find the root cause by identifying and monitoring substantial variables such as container and specimen type and collection personnel (Jones, 1997).

There are many causes of sample rejection, and these vary between institutions. But some criteria are common to all laboratories such as haemolysed, and clotted samples, insufficient and overfill, repetitive order and test not indicated. Generally, the causes of sample rejection include clotted and lysed samples or no sample received in the laboratory; samples are inadequately labelled or insufficient (Dale CJ., 2002). Clotted and haemolysed samples were the most common causes of sample rejection. Studies have linked these causes to inadequate or incorrect knowledge and skill at performing venipuncture and advocated the use of trained phlebotomists (Bolenius, 2013). Facilities using the service of laboratory-administered phlebotomists reported higher success rates than facilities with the non-laboratory-administered phlebotomist.

The cause of rejection is closely linked to the type of test and the location of the specimen. Gunnur, Pinar, and Akbiyik (2015) found that the ratio rejected specimens was higher in the emergency departments (ED) (40\%) compared to intensive care units (ICU) (30\%) and inpatient services (28\%). They have also identified that clotted samples (35\%) and insufficient samples (13\%) as the leading causes of rejection for coagulation tests, blood gas analyses and complete blood count (CBC) (Gunnur, 2015). Rooper, Carter, Hargrove, Hoffman, and Riedel (2017) also found ED to have the highest number of rejected samples, which contributed about $26.9 \%$ of all rejected samples. Their study showed that the highest rate of rejection was from neonatal ICU or NICU. The outpatient locations had $2.85 \%$ specimen rejection compared to inpatient locations (13.03\%) (Rooper et al.,2017). Stark et al. (2007) found that the specimens rejected from ED and inpatient services were 2-fold and 5-fold higher than for the outpatient services (Stark et al., 2007).

Rooper et al. (2017) proposed an algorithm to investigate the causes of specimen acceptability and target appropriate interventions. The intervention proposed was to design a cost-effective remediation plan targeted to the most common categories of the specimen and most influential factors for rejection (Rooper et al., 2017). Gunner et al. (2015) started to do enhanced documentation of rejected samples and targeted training for healthcare personnel at the departments with high rates of rejection.

Achieving a rejection rate of less than $1 \%$ would be quite a daunting task for our institution. Our annual rejection rate is always more than $2 \%$. This study aimed to reduce rejection rates and to improve our quality of care. The objectives of this study were to measure the rejection rate, to identify the causes of sample rejection and to suggest a few plans to mitigate it.

\subsection{Methodology}

This study was conducted at the Universiti Teknologi MARA (UiTM) Medical Specialist Centre (UiTMMSC), which caters for patients from the local community and referral cases from public healthcare centres. Our institution has the capacity of an 80-bedded hospital, offering services in mainly cardiology and cardiothoracic surgery. Other services include general surgery, respiratory, primary care, endocrinology, paediatric, obstetrics and gynaecology (excluding childbirth), orthopaedic, otorhinolaryngeal and emergency services. Our institution maintains two diagnostic laboratories, which are located in Sungai Buloh and Selayang. Both laboratories handle all the diagnostic test requests from the various clinical departments. The laboratory in Sungai Buloh operates 24 hours a day, whereas the other laboratory in Selayang only operates from 8 am till $5 \mathrm{pm}$. At the time of writing, we had no running Laboratory Information System. Thus, all data was extracted manually by designated staff.

This study only included sample rejection data from the Haematology (HM) and Transfusion Medicine Unit (TMU) from January to October 2019. The scope of tests performed in HM was complete blood count with white blood cell differentials(CBC-Diff), reticulocyte count, erythrocyte sedimentation rate (ESR), full blood picture, prothrombin time, activated partial thromboplastin time, thrombin time and D-Dimer. The haematoanalyser and haemostasis analyser used in our laboratory were Sysmex XN-550 and Stago STA Compact Max.

The laboratory rejection form was used to identify the cause for rejection. The medical laboratory technologists (MLT) were trained to assess sample integrity based on the criteria available in the rejection form, before running them on the machines. They were also able to add other causes of rejection, which were not mentioned in the criteria in the 'Other' box. The data from haematology and transfusion medicine sections were analysed exclusively and compiled according to the reasons (Table 1) and the location of the rejected specimens. However, we did not collect rejection data based on the type of test. All specimens were processed according to the Standard Operating Procedures in-use at the laboratories. Analysis of data was performed using Microsoft Excel and SPSS software. 
Table 1: Criteria for specimen rejection in the sample rejection form.

\begin{tabular}{ll}
\hline Defective label & Expired collection containers \\
\hline Missing label & Wrong collection containers \\
\hline Wrong label & Broken or cracked collection containers \\
\hline Incomplete request form & Insufficient specimen \\
\hline Haemolysed sample & No specimen received (only request form received) \\
\hline Lipaemic sample & Improper transportation method (specify:) \\
\hline Icteric sample & Temperature not maintained \\
\hline Clotted sample & Repetitive test \\
\hline Test not indicated & Test not offered \\
\hline Others: state & \\
\hline
\end{tabular}

\subsection{Findings}

The Haematology (HM) and Transfusion Medicine Unit (TMU) received a total of 17996 samples between January to October 2019. One thousand four hundred thirty samples were received at the TMU, and 16566 samples were received at the HM workstations. The HM and TMU rejected 560 (3.38\%) and 84 (5.7\%) samples, respectively. For comparison, both HM and TMU received a total of 20248 specimens in 2018. The rejection rates in 2018 were $2.78 \%$ for $\mathrm{HM}$ and $5.56 \%$ for TMU. The rejection rates for both years were above the target rate set by the MSQH, which is less than $1 \%$. The 2019 rejection rate was increased by $0.6 \%$ for HM compared to the previous year. The rejection rate for TMU was excessively high for both years (Table 2).

Table 2: Comparison between the rejection rates for Haematology and Transfusion Medicine Unit in 2018 and 2019.

\begin{tabular}{|l|c|c|c|c|}
\hline & \multicolumn{2}{|c|}{ Haematology (HM) } & \multicolumn{2}{c|}{ Transfusion Medicine (TMU) } \\
\hline & 2018 & Jan- Oct 2019 & 2018 & Jan- Oct 2019 \\
\hline Total samples received & 18596 & 16566 & 1652 & 1430 \\
\hline Rejected samples & 518 & 560 & 92 & 84 \\
\hline Percentage \% of rejection & 2.8 & 3.38 & 5.56 & 5.87 \\
\hline
\end{tabular}

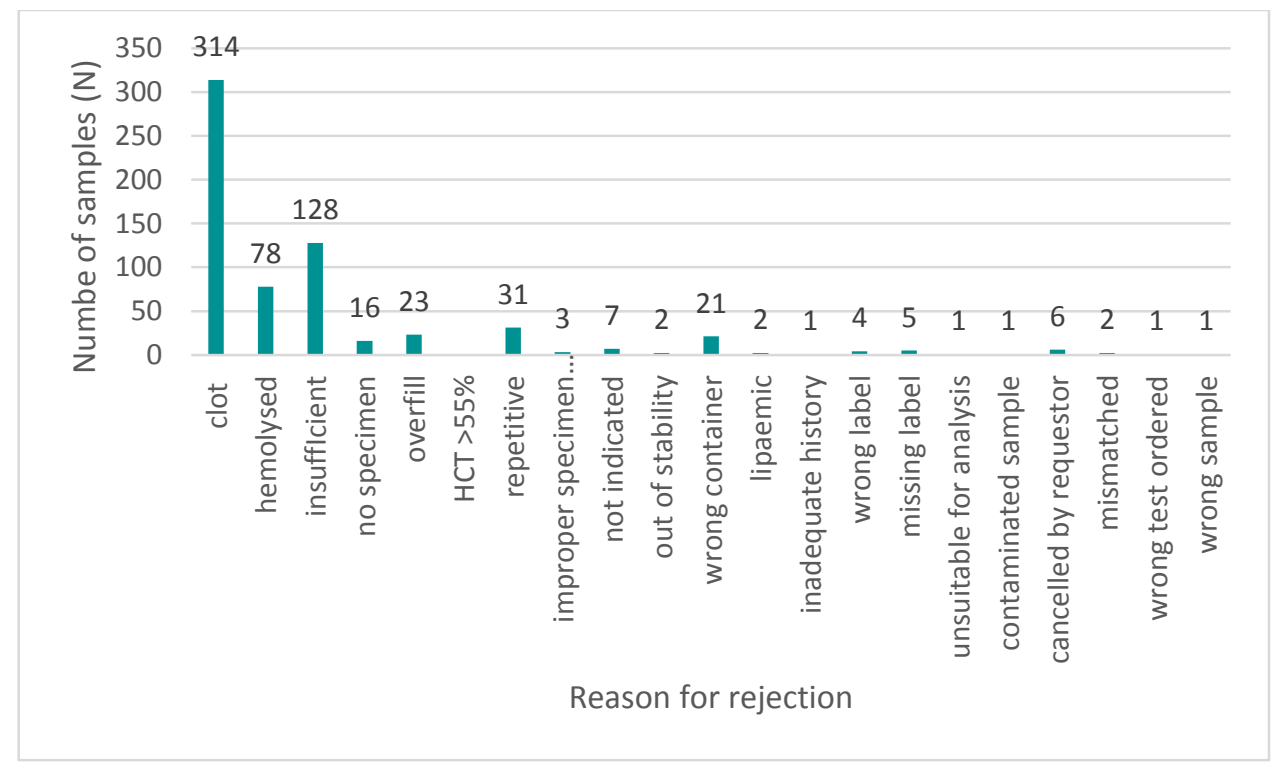

Figure 1: The reasons of sample rejection for Haematology (HM)

Clotted samples were found to be the leading cause of rejection for HM with a total of 314 , which constitute $56 \%$ of all rejected samples. This was followed by insufficient (22.9\%) and hemolyzed (13.9\%) samples. Twenty-three samples were rejected because they were overfilled. Thirty-one samples were rejected because of repetitive order, and 21 samples were rejected because of wrong containers. About seven samples were rejected because the tests were not indicated, and six requests were cancelled by the ordering doctors. 


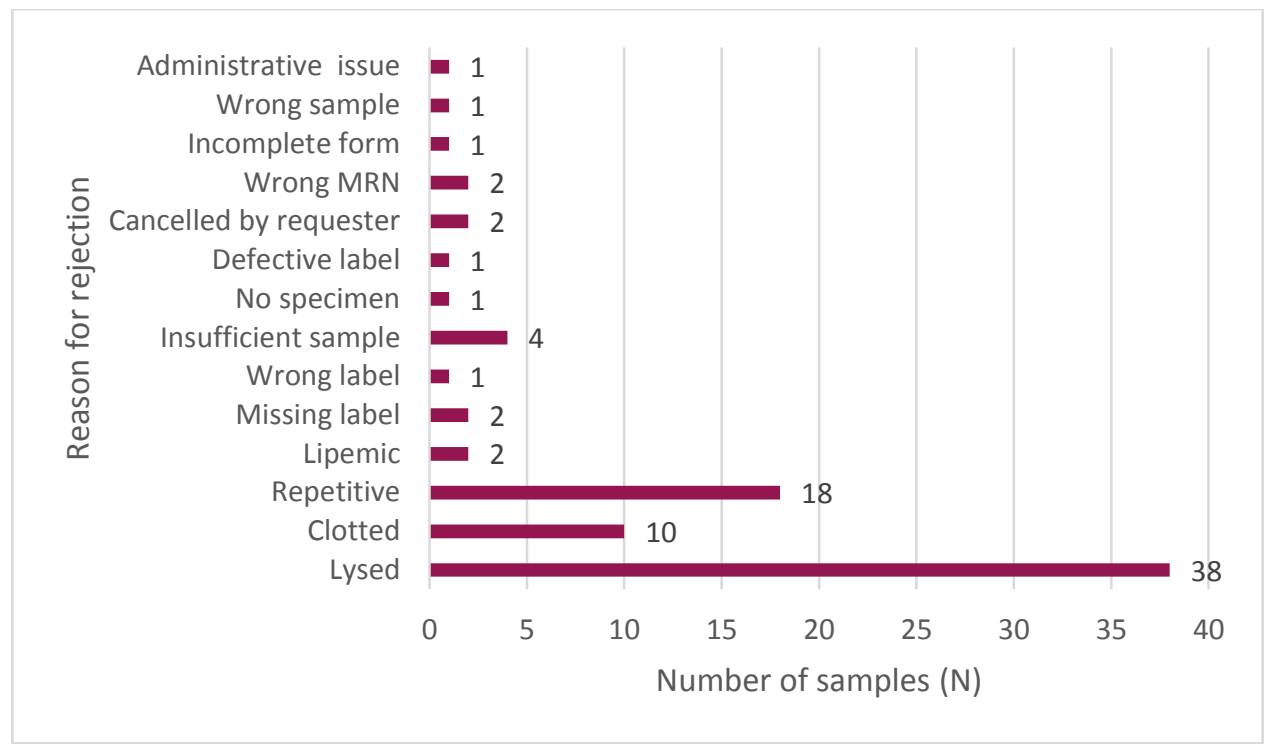

Figure 2: The reasons of sample rejection for Transfusion Medicine Unit (TMU)

For TMU, the most common cause of rejection was due to lysed (or haemolysed) samples, which constitute $45 \%$ of all rejected samples. Repetitive test order and clotted samples contributed $21.4 \%$ and $11.9 \%$, to the total number of rejection. 18 samples were rejected because of other causes such as administrative issue, wrong sample, incomplete form, wrong medical registration number (MRN), defective label, test cancelled by the requestor, no specimen, missing label and lipemic sample.

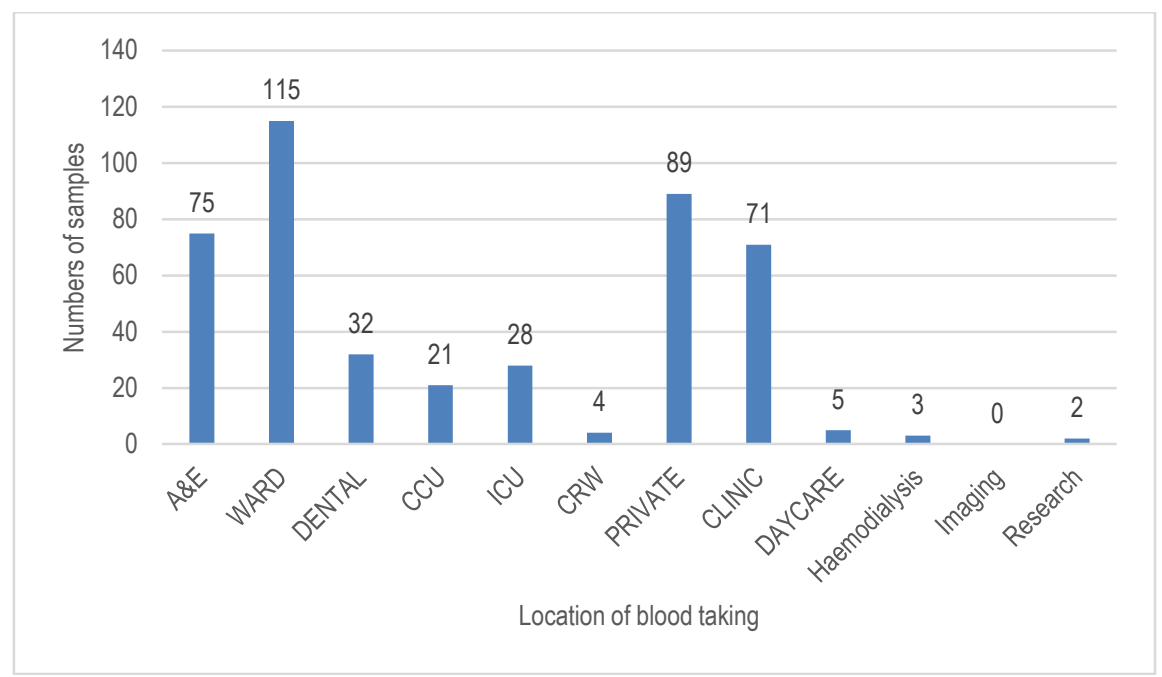

Figure 3: The location of the rejected samples for HM

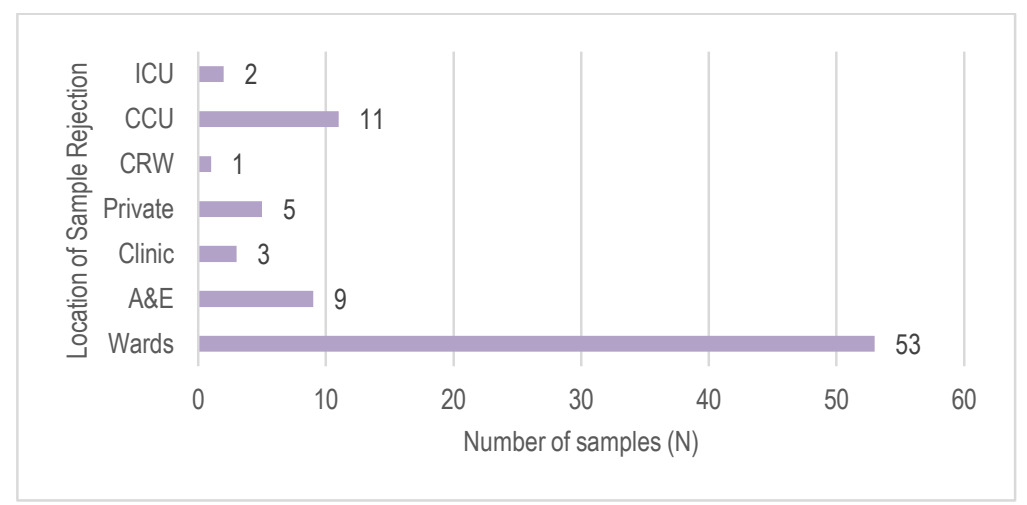

Figure 4: The location of the rejected samples for TMU 
Analysis of the locations of blood taking showed that most of the rejected samples in HM and TMU came from the wards. The HM rejected $20.5 \%$ of samples from the wards, while TMU rejected about $63 \%$ of samples (Figure 3 and 4 ). For HM, the private ward and clinic contributed about $15.9 \%$ of the rejected samples followed by the emergency department with $13.4 \%$. Other sites such as dental, coronary care unit (CCU) and Intensive Care Unit (ICU) each contributed to 32, 21 and 28 rejected samples. Coronary resuscitation ward, daycare, haemodialysis centre and research contributed less than 10 of the rejected samples.

The TMU rejected 11 samples from the CCU, and nine samples were from the A\&E. The ICU, CRW, clinic and private service contributed less than 10 of the total rejected samples.

When causes of rejection and sites of blood taking were analysed together, the most common cause of rejection in the HM was clotted sample, and most of these came from the wards. In comparison, the most common cause of rejection by TMU was haemolysed samples, and most of the rejected samples came from the wards.

Table 3: The monthly rejection rate for $\mathrm{HM}$.

\begin{tabular}{cccc} 
Months & Total samples & Total rejected samples & \% rejected samples \\
\hline Jan-19 & 1860 & 56 & 3.0 \\
\hline Feb-19 & 1527 & 44 & 2.9 \\
\hline Mar-19 & 1597 & 40 & 2.5 \\
\hline Apr-19 & 1690 & 75 & 4.4 \\
\hline May-19 & 1592 & 52 & 3.3 \\
\hline Jun-19 & 1194 & 34 & 2.8 \\
\hline Jul-19 & 1823 & 67 & 3.7 \\
\hline Aug-19 & 1867 & 80 & 4.3 \\
\hline Sep-19 & 1539 & 46 & 3.0 \\
\hline Oct-19 & 1877 & 66 & 3.5 \\
\hline
\end{tabular}

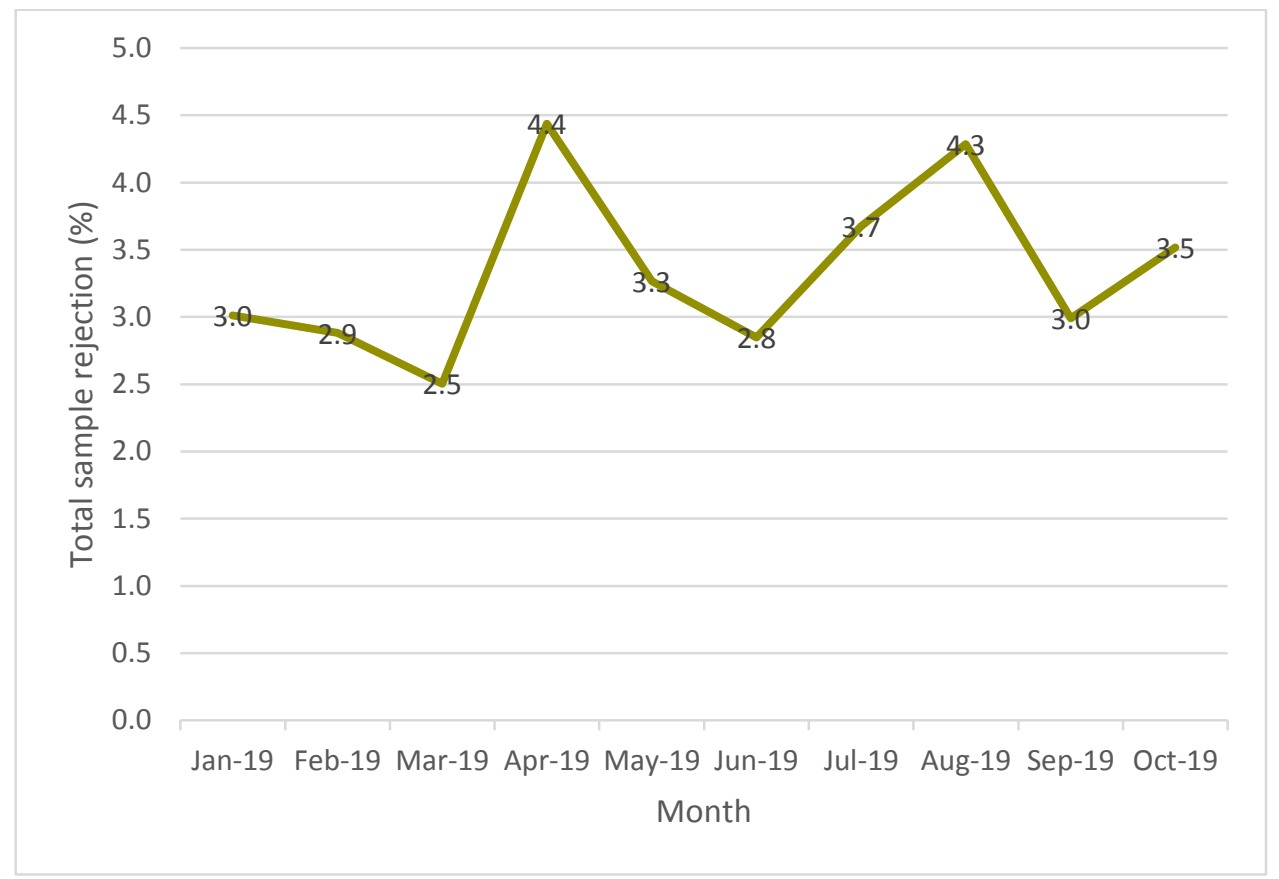

Figure 5: Monthly rejection rates.

The monthly rejection rates for $\mathrm{HM}$ fluctuated between $2.5 \%$ and $4.4 \%$. The highest rejection rates were in April, August and October 2019, whereas the lowest rejection rates were in March, June and September 2019.

\subsection{Discussion}

Our rejection rates were more than $1 \%$ for HM and TMU based on the analysis done using data from 2018 till October 2019, which is much higher than the recommended rate set in the $2017 \mathrm{MSQH}$ recommendation. Many studies reported a rejection rate of less than 
$1 \%$, but there were not as many studies reported a similar rate as ours. For example, a study done in Turkey reported a $2.7 \%$ rejection rate, in which clotted samples contributed $55.8 \%$ of total rejection (Lay, 2014). Another study done in India reported a rejection rate of $1.54 \%$, in which haemolysis was the leading cause of rejection (Atay, 2014). Most of the studies with higher rejection rates focused on documenting the causes of rejection. There was hardly any meta-analysis of the root causes of rejection and a comprehensive way to reduce the rates. Many studies were carried out in an integrated laboratory where the samples were received biochemistry and haematology tests.

There are many criteria used for sample rejection, and the criteria vary between institutions. We had specified about 21 criteria in our sample rejection form. However, we also allowed the medical technologists to state other causes of rejection if none of the options is suitable. The causes which were not included in the sample rejection form are inadequate history, missing label, wrong label, test cancelled by the requestor, mismatched samples, wrong test ordered and wrong sample. Some of the criteria were redundant and can be included in any one of the criteria in the form. However, that would depend on the judgement of the medical laboratory technologists in-charge of receiving the samples. Thus, the number of causes of sample rejection for both HM and TMU was not the same. All rejected samples were captured according to causes and location (origin) of the samples. Retrospectively, this could have been avoided if compilation and analysis of the rejected samples were done promptly at the end of every month. The availability of a reliable laboratory information system would ease the process.

This study found that the most common causes of sample rejection were due to sample clot and haemolysis, which is reflected in the findings in other studies (Rooper, 2017). Blood clot or fibrin often interfere with the interpretation of results by automatic analysers. For example, a blood clot can cause false thrombocytopenia, in which case the platelet count cannot be released. Fibrin can be readily seen under the microscope. Direct inspection of the sample before machine analysis can also detect fibrin clot in the tube. Haemolysed sample can cause spurious hyperkalemia. This should not be mistaken for in vivo haemolysis that could be caused by an actual pathological condition such as intravascular haemolysis. Haemolysed sample can also interfere in the interpretation of tests in the TMU and could cause wrong results to be released. In another study, Cao et al. reported contamination by IV fluid or TPN solution as being the most common cause of rejection, followed by inappropriate collection container/tube and quantity not sufficient as the second and third most frequent cause of rejection (Cao, 2016). These include prevention of prolonged venous stasis, collection of non-hemolyzed specimens, the order of draw, and appropriate filling and mixing of the primary collection tubes.

Samples received from the wards contributed most of the rejected samples compared to other locations, such as the emergency department (A\&E) and the clinics. Blood samples in the wards were mainly taken by the medical officers, compared to the A\&E and clinics, where venipunctures were mostly performed by the medical assistants and phlebotomists. This finding could reflect the blood taking skill or technique used by the staff. This is also reflected in the number of rejections for TMU, which rejected more samples than HM. Other explanation could be that inpatients were sicker compared to outpatients and made blood-taking more difficult, in which prolonged venous stasis can lead to blood clot formation. Overall, there were fewer samples received by the laboratory from ICU, CCU and CRW. The number of samples rejected by both HM and TMU reflected these numbers. The presence of a robust link between the hospital information system (HIS) and the laboratory information system (LIS) could have reduced sample rejection because of repetitive order. Insufficient sample contributed to the causes of sample rejection from these sites, especially in long-staying patients who had multiple venipunctures.

This study highlighted the need to identify the causes of rejection and subsequently formulate a plan to mitigate the problem. There were many suggestions on methods to reduce the number of rejected samples. Education and retraining of staffs involved in venipuncture are important to refresh the knowledge and skill of the staffs. Knowledge of order of draw for sample tubes is vital to prevent contamination with additives (Lippi, 2012). For example, sample for coagulation (light blue top) should be collected after blood culture bottle or no additive tubes and before any other type of additive tube. Samples should be gently, not vigorously, inverted at least one to two times immediately after blood collection. Although educational interventions were shown to improve the rejection rates, the sustainability of the benefit remains doubtful for a few reasons. A regular session on education would cause lack of staffs for a few hours at the clinics or other healthcare locations. It could also interfere with clinical workflow and indirectly affect a patient's quality of care (Rooper, 2017). We are aware of these problems and would organise similar regular educational intervention involving small groups of participants, which would minimise the interruption in workflow due to lack of staffs.

In our study, the clinics had a lower rejection rate compared to the wards, but the number was quite high. This finding is similar to a study done by Rooper et al. (2017), which showed that the outpatient locations had a lower rejection rate than inpatient locations, which could be because there were not enough phlebotomists to cater for all blood taking requests from the clinics. At our institution, there were only two trained phlebotomists. The staff nurses had formal training before they were assigned at the clinics. Their service started in 2017 and was only limited to nearby clinics. This problem could be mitigated by training or hiring more phlebotomists to cater not only to clinics but to the wards as well. An interdisciplinary collaboration between the laboratory and the clinical departments in the form of education as well as periodic assessment can be done to reduce and maintain the rejection rate at an acceptable range.

Other measures of improvement include the use of better phlebotomy equipment such as using straight needles rather than butterfly devices or syringes. Some studies suggested the use of larger needles, i.e. size 19 to 21 gauge; and primary vacuum tubes to prevent haemolysed sample. We excluded the data of rejection based on the type of test. The information on the type of test would increase our understanding of the cause of rejection because some tests require different blood taking technique. For example, a neonate has smaller veins and thus requires less volume for analysis compared to adults. Technically, it is more difficult to get blood from these patients, and thus, more rejection caused by clotted samples was seen in NICU (Rooper, 2017). We did not have any neonatal patients at our facility, but we do have many patients requiring coagulation tests, which requires specific precaution. Some studies suggested reducing the use of tourniquet when taking blood for haemostasis tests and one study suggested limiting the time to less than a minute. One study 
suggested using only venipuncture for blood taking instead of intravenous catheters. Giavarina and Lippi (2017) suggested the implementation of a simple checklist, which includes verification of blood collection devices, patient preparation and sampling techniques, to enhance sample quality and reduce some preanalytical errors (Giovarina, 2017).

The sample rejection rates were noted to fluctuate around the target at specific patient care location within the hospital (Rooper, 2017). We found this to occur in our study as well, although the reason for the fluctuation was unclear.

\subsection{Conclusion and Recommendations}

In conclusion, it is difficult for us to achieve the recommended rejection rate. Based on this study, we decided to set a realistic target of $1.5 \%$ rejection rate for $\mathrm{HM}$ and less than $2 \%$ for TMU. This target may seem impossible for us at the moment, but we are quite optimistic about it. From this study, we were able to identify the most common causes of sample rejection in our laboratories and would use that knowledge to formulate a comprehensive plan to mitigate them. We found many recommendations in the literature on ways to reduce rejection rates. However, more studies are needed to assess the usefulness of the recommendations.

\section{Acknowledgements}

We would like to acknowledge the staffs of Department of Pathology and the Faculty of Medicine Universiti Teknologi MARA for their assistance and support in this study.

\section{References}

Alsina, M. J., Álvarez, V., Barba, N., Bullich, S., Cortés, M., Escoda, l., \& Martínez-Brú, C. (2008). Preanalytical quality control program - An overview of results (20012005 summary). Clinical Chemistry and Laboratory Medicine, 46(6), 849-854. https://doi.org/10.1515/CCLM.2008.168

Atay, A., Demir, L., Cuhadar, S., Saglam, G., Unal, H., Aksun, S., ... Sutcu, R. (2014). Clinical biochemistry laboratory rejection rates due to various types of preanalytical errors. Biochemia Medica, 24(3), 376-382. https://doi.org/10.11613?BM.2014.040.

Bölenius, K., Söderberg, J., Hultdin, J., Lindkvist, M., Brulin, C., \& Grankvist, K. (2013). Minor improvement of venous blood specimen collection practices in primary health care after a large-scale educational intervention. Clinical Chemistry and Laboratory Medicine, 51(2), 303-310. https://doi.org/10.1515/cclm-2012-0159.

Bonini, P., Plebani, M., Ceriotti, F., \& Rubboli, F. (2002). Errors in laboratory medicine. Clinical Chemistry. https://doi.org/10.1093/clinchem/48.5.69.

Cao, L., Chen, M., Phipps, R. A., Del Guidice, R. E., Handy, B. C., Wagar, E. A., \& Meng, Q. H. (2016). Causes and impact of specimen rejection in a clinical chemistry laboratory. Clinica Chimica Acta, 458, 154-158. https://doi.org/10.1016/j.cca.2016.05.003.

Dale, J. C., \& Novis, D. A. (2002). Outpatient phlebotomy success and reasons for specimen rejection: A Q-Probes study. Archives of Pathology and Laboratory Medicine, 126(4), 416-419. https://doi.org/10.1043/0003-9985(2002)126<0416:OPSARF>2.0.CO;2

Giavarina, D., \& Lippi, G. (2017). Blood venous collection: Recommendations overview and a checklist to improve quality. Clinical Biochemistry. Elsevier Inc. https://doi.org/10.1016/j.clinbiochem.2017.02.021.

Gunnur Dikmen, Z., Pinar, A., \& Akbiyik, F. (2015) Specimen rejection in laboratory medicine: Necessary rejection for patient safety? Biochemica Medica, 25(3), 377-385. https://doi.org/10.11613/BM.2015.037.

Jacobsz, L. A., Zemlin, A. E., Roos, M. J., \& Erasmus, R. T. (2011). Chemistry and haematology sample rejection and clinical impact in a tertiary laboratory in Cape Town. Clinical Chemistry and Laboratory Medicine, 49(12), 2047-2050. https://doi.org/10.1515/CCLM.2011.743

Jones, B. A., Calam, R. R., \& Howanitz, P. J. (1997). Chemistry specimen acceptability: A College of American Pathologists Q- Probes study of 453 laboratories. Archives of Pathology and Laboratory Medicine, 121(1), 19-26.

Karcher, D. S., \& Lehman, C. M. (2014). Clinical consequences of specimen rejection: A college of american pathologists Q-probes analysis of 78 clinical laboratories. Archives of Pathology and Laboratory Medicine, 138(8), 1003-1008. https://doi.org/10.5858/arpa.2013-0331-CP

Lippi, G., Salvagno, G., Montagnana, M., Lima-Oliveira, G., Guidi, G., \& Favaloro, E. J. (2012). Quality standards for sample collection in coagulation testing. Seminars in Thrombosis and Hemostasis, 38(6), 565-575. https://doi.org/10.1055/s-0032-1315961.

Malaysian Society for Quality in Health. (2017). Performance Indicators - MSQH Hospital Accreditation Standards 5th Edition. Malaysian Society for Quality in Health.

Stark, A., Jones, B. A., Chapman, D., Well, K., Krajenta, R., Meier, F. A., \& Zarbo, R. J. (2007). Clinical laboratory specimen rejection - Association with the site of patient care and patients' characteristics: Findings from a single health care organization. Archives of Pathology and Laboratory Medicine, $131(4), 588-592$. https://doi.org/10.1043/1543-2165(2007)131[588:CLSRWT]2.0.C0;2.

Rooper, L., Carter, J., Hargrove, J., Hoffmann, S., \& Riedel, S. (2017). Targeting rejection: Analysis of specimen acceptability and rejection, and framework for identifying interventions in a single tertiary healthcare facility. Journal of Clinical Laboratory Analysis, 31(3). https://doi.org/10.1002/jcla.22060.

Sinici Lay, I., Pinar, A., \& Akbiyik, F. (2014). Classification of reasons for rejection of biological specimens based on pre-preanalytical processes to identify quality indicators at a university hospital clinical laboratory in Turkey. Clinical Biochemistry, 47(12), 1002-1005. https://doi.org/10.1016/j.clinbiochem.2014.04.024. 